Revista Brasileira de Agricultura Irrigada v.13, no.3, p. 3390 - 3401, 2019

ISSN 1982-7679 (On-line)

Fortaleza, CE, INOVAGRI - http://www.inovagri.org.br

DOI: $10.7127 /$ rbai.v13n301007

Protocolo 1007.19 - 27/08/2018 Aprovado em 19/06/2019

\title{
ANÁLISE PROBABILÍSTICA DA TEMPERATURA MÁXIMA EM URUGUAIANA, RS
}

\author{
Roger Vinicius de Castro Ferreira ${ }^{1}$, Gilberto Rodrigues Liska ${ }^{2}$
}

\begin{abstract}
RESUMO
A teoria dos valores extremos desempenha um papel fundamental na modelagem de eventos associados a probabilidades baixas ou eventos raros. Os modelos probabilísticos baseados nesta teoria visam predizer, a partir de um conjunto de valores máximos de um processo ambiental registrado num período relativamente curto (30 anos, por exemplo), os valores máximos esperados em um período maior (50, 100 ou mais anos). Para o caso específico das temperaturas, são de grande utilidade, por exemplo, no planejamento de projetos agrícolas. Este trabalho teve por objetivo empregar as distribuições generalizada de valores extremos (GVE) e Gumbel aos dados de temperatura máxima mensal registradas durante um período de 64 anos (1951 a 2015) em Uruguaiana, Estado do Rio Grande do Sul. Para a estimação dos parâmetros dessa distribuição, foi utilizado o método da máxima verossimilhança. $\mathrm{O}$ ajuste aos dados foi avaliado por meio dos gráficos de envelopes simulado e do teste de Kolmogorov-Smirnov. A distribuição GVE se ajustou aos dados em todos os meses do ano e, em particular, nos meses de março, outubro e novembro a distribuição Gumbel é mais adequada. Nos meses de maio a agosto ocorreram as menores temperaturas previstas para todos os níveis de retorno, e nos meses de dezembro a fevereiro ocorreram as maiores temperaturas previstas para todos os níveis de retorno calculados.
\end{abstract}

Palavras-chave: variáveis meteorológicas, distribuição Gumbel, eventos extremos, climatologia.

\section{PROBABILISTIC ANALYSIS OF MAXIMUM TEMPERATURE IN URUGUAIANA, RS.}

\begin{abstract}
The extreme value theory plays a fundamental role in modeling events associated with low probabilities or rare events. The probabilistic models based on this theory aim to predict, from a set of maximum values of an environmental process registered in a relatively short period (30 years, for example), the maximum values expected in a longer period of time (50,100 or more years). For the specific case of temperatures, they are very useful, for example, in the planning of agricultural projects. The objective of this work was to evaluate the generalized extreme value distributions (GEV) and

\footnotetext{
${ }^{1}$ Discente da graduação em Engenharia Cartográfica e de Agrimensura, Universidade Federal do Pampa, campus Itaqui, Rua Luiz Joaquim de Sá Britto - s/n - Bairro Promorar, CEP: 97650-000, e-mail: rogervcf@ hotmail.com

${ }^{2}$ Docente da Universidade Federal do Pampa, campus Itaqui, Rua Luiz Joaquim de Sá Britto - s/n - Bairro Promorar, CEP: 97650-000, e-mail: gilbertoliska@unipampa.edu.br
} 
Gumbel to the monthly maximum temperature data recorded during a period of 64 years (1951 to 2015) in Uruguaiana, Rio Grande do Sul. For proving the parameters estimates of these distributions, the maximum likelihood method was used. The fit to the data was evaluated by means of the simulated envelope plots and Kolmogorov-Smirnov test. The GEV distribution has adjusted to all months of the year and in particular in the months of March, October and November the Gumbel distribution is more adequate. During the months of May to August, the lowest expected temperatures occurred for all return levels, and in the months of December to February occurred the highest temperatures predicted for all return levels calculated.

Keywords: meteorological variables, Gumbel distribution, extremes values, climatology.

\section{INTRODUÇÃO}

Nosso planeta seguiu uma evolução desde sua origem, passando por transformações significativas em sua atmosfera. Algumas componentes críticas do funcionamento básico do sistema terrestre estão se modificando, e entre elas estão o clima e a composição atmosférica.

O desenvolvimento da agricultura e o inicio da revolução industrial levaram a um explosivo crescimento populacional, que fez pressões importantes sobre os recursos naturais do planeta (ARTAXO, 2014). No cenário climatológico da agricultura, existem fatores que influenciam diretamente sob o desenvolvimento de animais e plantas, e estão sendo influenciados pela ação humana, fato que pode colocar em risco muitos projetos agrícolas (AYOADE, 2006). O dimensionamento do risco de ocorrência de temperaturas máximas extremas mensais e anual são informações relevantes para o planejamento e a tomada de decisões de agricultores e órgãos governamentais, pois podem diminuir os impactos causados pelos níveis máximos de temperatura (ASTOLPHO et al., 2004). Em seus níveis mais elevados, é um dos elementos climáticos de maior importância para $\mathrm{o}$ crescimento, o desenvolvimento e a produtividade de culturas como o arroz, a soja e o milho (WALTER et al., 2014).

No estudo da adaptabilidade e comportamento das culturas agrícolas é importante o conhecimento do comportamento de variáveis climáticas. A relação clima-planta é caracterizada e mensurada por meio da magnitude dessas variáveis e da resposta da planta ao meio ambiente. $\mathrm{O}$ estudo da disponibilidade térmica do ambiente às culturas agrícolas, geralmente, deve ser realizado com a temperatura em suas magnitudes mais importantes, médias, máximas ou mínimas (CARGNELUTTI FILHO et al., 2006). Os efeitos da temperatura máxima do ar nas culturas podem ser devidos a influência na duração da fase de enchimento de grãos, taxa de respiração e na taxa de expansão foliar. Vale salientar que, todos esses efeitos podem provocar redução na produtividade das culturas (COSTA; SEDIYAMA, 1999). Embora a teoria probabilística de valores extremos tenha sido desenvolvida há muito tempo, a modelagem estatística de extremos ainda permanece como assunto ativo de pesquisas, dado seu importante papel nos projetos e gerenciamento de recursos hídricos, especialmente num contexto de mudanças climáticas (ARAÚJO et al., 2010). A Teoria de valores extremos (TVE) desempenha um papel fundamental em estudos relacionados a medições físicas, sendo aplicada com a finalidade de descrever o comportamento de eventos raros e tem sido aplicada com sucesso no tratamento estatístico de dados meteorológicos, tais como temperaturas máximas e mínimas, ventos máximos e mínimos, entre outros (BEIJO; AVELAR, 2011; THOMAS et al., 2016).

A utilização de funções de distribuição de probabilidade requer o uso de testes para avaliar a adaptação dos dados ou da série de dados às funções. Esses testes são conhecidos como testes de aderência e sua real função é verificar a adequação de uma distribuição hipotética aos dados (ARAÚJO et al., 2010). Em relação aos extremos de temperatura, não 
existe um consenso sobre distribuições preferenciais. Sansigolo (2008) constatou que a distribuição Gumbel é adequada para estudos de extremos de temperatura máxima, bem como Reis et al. (2017), que constataram que a distribuição Gumbel foi a que melhor se ajustou às temperaturas mínimas em alguns meses em Piracicaba, SP.

Tendo em vista a importância do conhecimento das possíveis temperaturas máximas, este trabalho teve como objetivo a aplicação da Teoria de Valores Extremos (TVE) aos dados de temperatura máxima de Uruguaiana, RS, visando obter a probabilidade de ocorrência mensal de temperaturas acima de $30,35,37,39,40,41,42$ e $45^{\circ} \mathrm{C}$ e estimar as temperaturas máximas para períodos de retorno de $5,10,30,50$ e 100 anos, uma vez que informações do tipo são de grande importância para adaptação aos impactos climáticos sobre a agricultura.

\section{MATERIAIS E MÉTODOS}

Os dados de temperatura foram obtidos no período de 1950 a 2016, de todos os meses dos respectivos anos em estudo, do município de Uruguaiana, RS, fornecidos pelo Banco de Dados Meteorológicos para Ensino e Pesquisa - BDMEP (INMET, 2018). As séries foram organizadas em períodos mensais e extraiu-se a temperatura máxima de cada mês, formando assim novas séries com os máximos mensais.

A TVE assegura a existência de uma distribuição assintótica não degenerada $F(x)$ para uma transformação linear de $M_{n}$ (COLES, 2001). Isto é, sequências numéricas apropriadas $a_{n}>0$ e $b_{n} \in \mathbb{R}$, de tal modo que:

$$
P\left(\frac{M_{n}-b_{n}}{a_{n}} \leq x\right) \rightarrow F(x) .
$$

Jenkison (1955) mostrou que os três tipos de distribuição de valores extremos (Gumbel, Fréchet e Weibull) poderiam ser representados em uma forma aramétrica única, denominada. Distribuição Generalizada de Valores Extremos (GVE) que possui distribuição de função acumulada definida pela equação 2 :

$$
F(x)=\exp \left\{-\left[1+\xi\left(\frac{x-\mu}{\sigma}\right)\right]^{-\frac{1}{\xi}}\right\}
$$

Definida no conjunto, em que os parâmetros satisfazem $-\infty<\mu<\infty, \sigma>0$ e $-\infty<$ $\xi<\infty$. O modelo consiste de três parâmetros: um parâmetro de locação, $\mu$; um parâmetro de escala, $\sigma$; e um parâmetro de forma, $\xi$. As famílias de distribuição Gumbel, Fréchet e
Weibull de valores extremos correspondem, aos casos, $\quad \xi=0, \quad \xi>0, \quad \xi<0$, respectivamente (HARTMANN et al., 2011).

Para o caso particular em que $\xi=0$ a função de distribuição acumulada Gumbel é definida pela equação 3:

$$
F(x)=\exp \left\{-\exp \left[-\left(\frac{x-\mu}{\sigma}\right)\right]\right\}
$$

Definida em $-\infty<x<\infty$, em que $\mu$ é o parâmetro de locação e $\sigma$ o parâmetro de escala, com $\mu \in \mathbb{R}$ e $\sigma>0$. Os parâmetros dos modelos (2) e (3) são desconhecidos e precisam ser estimados. Para tal, foi utilizado o método da máxima verossimilhança, cuja função de verossimilhança com base em uma amostra aleatória é dada pela equação 4 :

$$
L\left(\Theta ; x_{1}, \ldots, x_{n}\right)=f\left(x_{1} ; \Theta\right) \times \ldots \times f\left(x_{n} ; \Theta\right)=\prod_{i=1}^{n} f\left(x_{i} ; \Theta\right)
$$


Que consiste em adotar como estimador a estatística que maximiza a probabilidade da amostra ter ocorrido, sob a hipótese de que $X_{1}$, $X_{2}, \ldots, X_{n}$ são variáveis aleatórias independentes e identicamente distribuídas (i.i.d.).

Esse pressuposto foi avaliado pelo teste de Ljung-Box e pelo teste de Aleatoriedade, conforme recomendação de Bautista et al. (2004). De acordo com Coles (2001), o parâmetro $\quad \xi$ pode ser testado se é estatisticamente nulo ou não. Para tal, foi utilizado o teste de razão de verossimilhanças $\left(T_{R V}\right)$, que consiste em comparar os máximos do logaritmo da função de verossimilhança das distribuições GVE (2) e Gumbel (3), $l\left(\Theta_{G V E}\right)$ e $l\left(\Theta_{G}\right)$, por meio da distribuição qui-quadrado. A estatística do teste é dada pela equação 5:

$$
T_{R V}=-2\left[l\left(\widehat{\Theta_{G}}\right)-l\left(\widehat{\Theta_{G V E}}\right)\right]=2\left[l\left(\widehat{\Theta_{G V E}}\right)-l\left(\widehat{\Theta_{G}}\right)\right]
$$

Em que $\widehat{\Theta_{G V E}}=(\mu, \sigma, \xi)$ e $\widehat{\Theta_{G}}=(\mu, \sigma)$ são vetores de estimativas de máxima verossimilhança das distribuições GVE e Gumbel, respectivamente.

Dessa forma, rejeita-se a hipótese nula de que $\xi=0$ se $T_{R V}$ for superior ao quantil da distribuição qui-quadrado com 1 grau de liberdade.

Os testes de aderência qui-quadrado, Kolmogorov-Smirnov, Lilliefors, ShapiroWilk, Cramer-von Mises, servem para comparar as probabilidades empíricas de uma variável com as probabilidades teóricas estimadas pela função de distribuição em teste, verificando se os valores da amostra podem ser provenientes de uma população com aquela distribuição (HARTMANN et al. 2011).

Para testar a suposição de que os máximos mensais de temperatura seguem uma distribuição GVE ou Gumbel (hipótese $H_{0}$ ), foi utilizado o teste de Kolmogorov-Smirnov, cuja estatística de teste $(D)$ é definida por:

$$
D=\max \left|F\left(X_{(i)}\right)-\widehat{F}\left(X_{(i)}\right)\right|, i=1,2, \ldots, n .
$$

Com $F\left(X_{(i)}\right)$ sendo a distribuição acumulada teórica da distribuição GVE ou Gumbel com suas estimativas obtidas e $\hat{F}\left(X_{(i)}\right)$ a distribuição acumulada empírica. De acordo com Quadros et al., (2011), a temperatura máxima provável $(X)$ par um determinado tempo de retorno $T$, pela distribuição GVE, pode ser determinada pela seguinte expressão:

$$
X=\hat{\mu}-\frac{\hat{\sigma}}{\hat{\xi}} \times\left[1-\ln \left(1-\frac{1}{T}\right)^{-\hat{\xi}}\right],
$$

Em que $\hat{\mu}$, $\hat{\sigma}$ e $\hat{\xi}$ são as estimativas de máxima verossimilhança. De maneira análoga, obtém-se o nível de retorno para a distribuição Gumbel, compreendendo o caso na Equação (7) em que $\xi=$ 0. Para realização das análises, testes de hipóteses e cálculo dos níveis de retorno, utilizou-se o software R (R CORE TEAM, 2017), juntamente com os pacotes evd (STHEPHENSON, 2002) e extRemes (GILLELAND; KATZ, 2016).

\section{RESULTADOS E DISCUSSÃO}

Os dados rearranjados em series de máximas mensais foram submetidos ao Teste de LjungBox, cuja hipótese nula $\left(H_{0}\right)$ é a independência dos máximos mensais de temperatura. Os resultados foram maiores que o nível de significância de 5\% ( $p$-valor > 0,05), concluindo que os dados são independentes. Esses resultados diferem dos resultados obtidos por Sartori et al. (2010), que ao analisarem as médias mensais de temperatura e umidade relativa do ar e os totais mensais de precipitação pluviométrica, do período de 1988-2007, concluíram que as observações das três variáveis climáticas apresentaram 
fortes dependência temporal. Convém ressaltar que a independência e aleatoriedade das observações são pressuposição básica da TVE e, caso isso não fosse atendido, as conclusões poderiam não serem válidas.

Camargo et al. (1993) e Astolpho et al. (2004), ao avaliarem o ajuste da distribuição Gumbel às series históricas de temperatura mínima de alguns municípios do estado de São Paulo, não informaram se a pressuposição de independência dos dados foi satisfeita. Em seguida foi feito o Teste de Aleatoriedade, cuja hipótese nula $\left(H_{0}\right)$ é a ausência de tendência dos máximos mensais de temperatura, e concluiu-se que os dados não apresentam tendência, uma vez que os $p$-valores são superiores a 5\% (Tabela 1). Séries temporais longas possibilitam melhores ajustes e verificações, mas por outro lado podem estar contaminadas pela presença de tendências e ciclos de longo prazo, que devem ser previamente analisados (SANSIGOLO, 2008; SALVIANO et al., 2016).

Tabela 1. $P$-valores dos testes de Ljung-Box sob o pressuposto de independência (Ljung-Box) e de Aleatoriedade (Runs-test), aplicado aos dados de temperatura máxima no município de Uruguaiana.

\begin{tabular}{ccc}
\hline Meses & Ljung-Box $(p$-valor $)$ & Runs-test $(p$-valor $)$ \\
\hline Janeiro & 0,1759 & 0,2371 \\
Fevereiro & 0,9117 & 0,9035 \\
Março & 0,4893 & 0,1756 \\
Abril & 0,1092 & 0,0671 \\
Maio & 0,2224 & 0,1359 \\
Junho & 0,6005 & 0,7655 \\
Julho & 0,5932 & 0,7603 \\
Agosto & 0,3704 & 0,7603 \\
Setembro & 0,6392 & 0,4672 \\
Outubro & 0,8297 & 0,8827 \\
Novembro & 0,7584 & 0,8827 \\
Dezembro & 0,2830 & 0,9035
\end{tabular}

Fonte: Autoria própria, 2019.

Nota: $p$-valor $<\alpha$, rejeita-se $H_{0}$, em que $\alpha$ denota o nível de significância do teste.

Os resultados do teste KolmogorovSmirnov indicam que em todos os meses em estudo as distribuições GVE e Gumbel se ajustaram aos dados de temperatura máxima, uma vez que o $p$-valor em todos os meses são superiores a 0,05. Como o teste Kolmogorov-
Smirnov é insensível nos extremos da distribuição e testes alternativos satisfatórios não existem, gráficos QQ, mostrados na Figura 1, foram complementarmente usados para avaliar o comportamento das distribuições nos seus extremos. 
Ferreira et al.

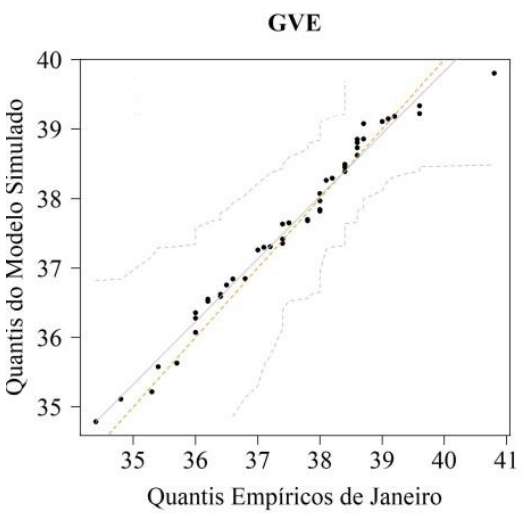

GVE

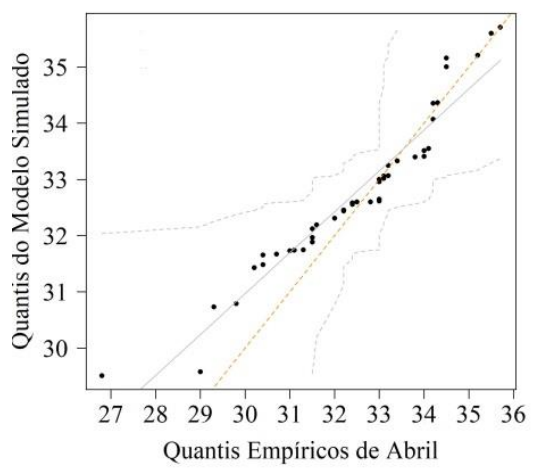

GVE

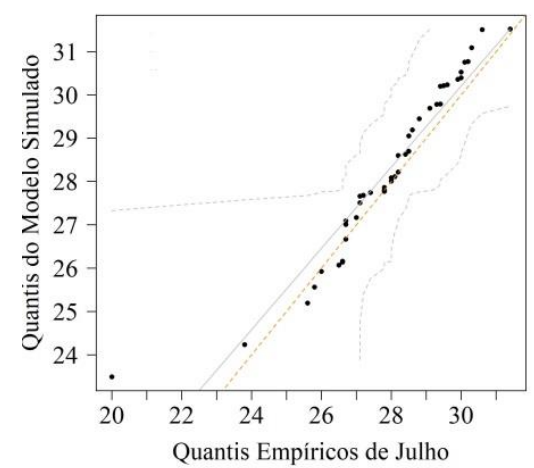

Gumbel

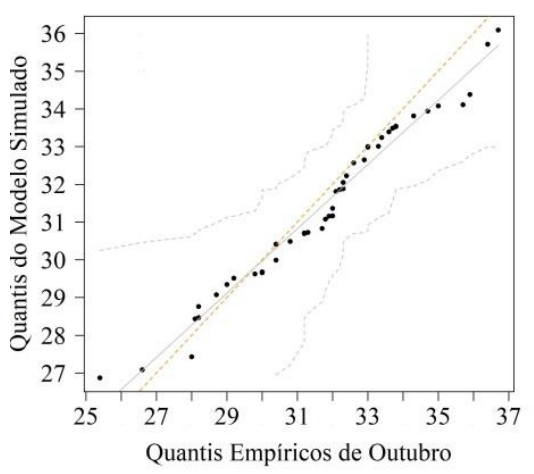

Linha 1-1

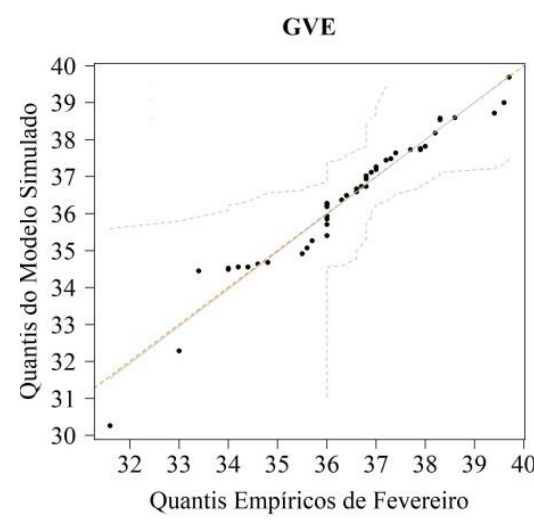

GVE

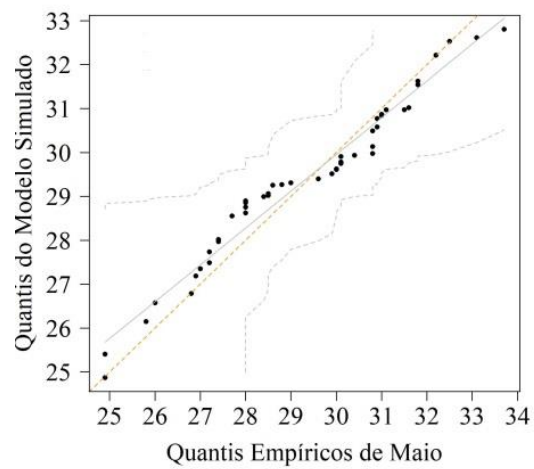

Gumbel

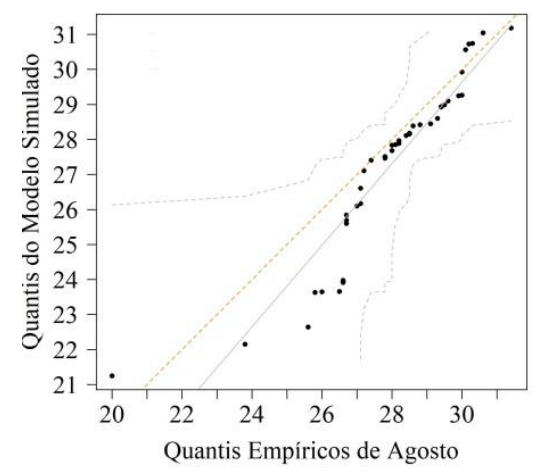

Gumbel

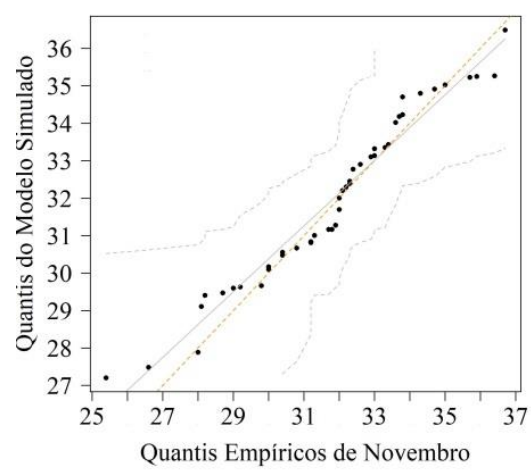

Linha de regressão

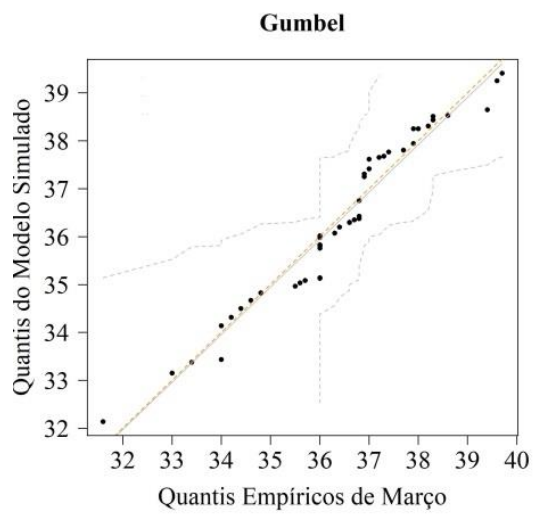

GVE

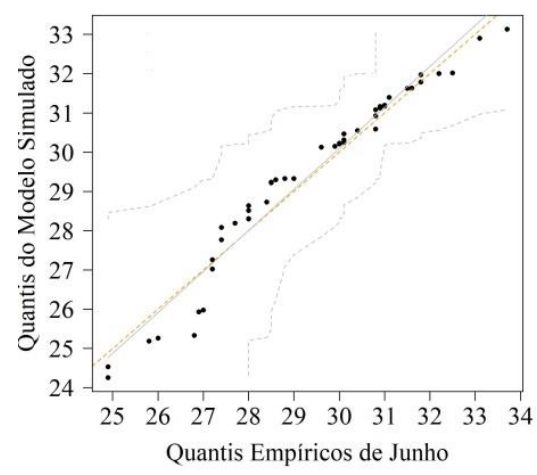

GVE

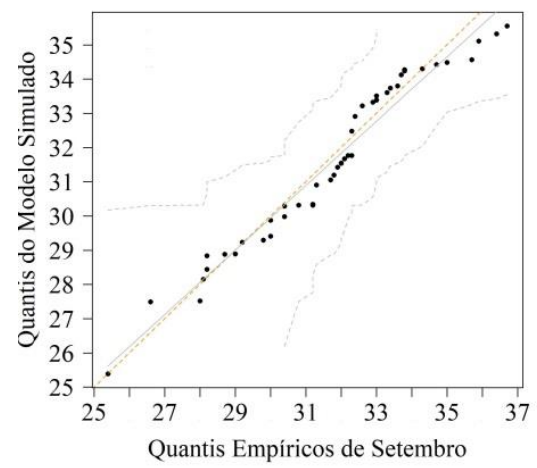

GVE

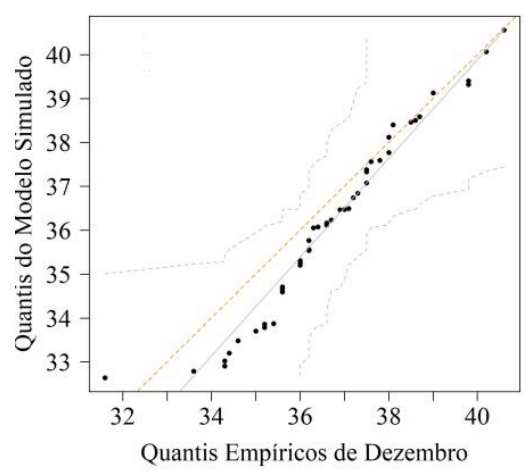

Intervalo de confiança de $95 \%$

Figura 1. Gráfico de envelope simulado para cada mês com a respectiva distribuição de probabilidade de acordo com a Tabela 2, para os dados de temperatura máxima no período de 1961 a 2016 em Uruguaiana-RS. A borda tracejada em cinza indica um intervalo de confiança de 95\% construído por simulação de Monte Carlo. 
Os gráficos de quantil-quantil para o diagnóstico da distribuição GVE e Gumbel comparam os quantis da distribuição teórica com os quantis da distribuição ajustada. O ajuste ocorre se o par ordenado formado por esses quantis estiverem próximos da linha 1-1, ou seja, o quantil estimado pela distribuição proposta é igual (ou próximo) do quantil empírico. Contudo, a decisão de que o modelo proposto se ajusta aos dados por essa forma pode ser subjetiva e, para tal, foi construído o intervalo de confiança desses mesmos quantis.

Para construção das bandas de confiança, sob a hipótese de que modelo se ajusta aos dados, foram simulados máximos mensais com as estimativas dos parâmetros da Tabela 1 e em seguida foram calculados os quantis teóricos.
Após um número de simulações, para cada quantil empírico, obtém-se o máximo e o mínimo dos quantis simulados. As bandas formadas pelos máximos e mínimos simulados constituem as bandas de confiança e o gráfico resultante também é chamado de gráfico de envelope simulado (GILLELAND; KATZ, 2016). Dessa forma, o ajuste do modelo é satisfatório se a grande maioria dos pares ordenados estiverem contidos nas bandas de confiança construídas por simulação de Monte Carlo com 95\% de confiança. Assim, os gráficos da Figura 1 corroboram o ajuste das distribuições na Tabela 2. De acordo com Sansigolo (2008), tem-se uma maior precisão no ajuste da distribuição GVE quando se utiliza uma série histórica igual ou superior a 30 anos.

Tabela 2. Resultados das estimativas dos parâmetros das distribuições (GVE e Gumbel), testes de Kolmogorov-Smirnov (KS) para os dados de temperatura máxima no município de Uruguaiana.

\begin{tabular}{|c|c|c|c|c|c|c|c|c|}
\hline \multirow{2}{*}{ Meses } & \multirow{2}{*}{ Distribuição } & \multicolumn{3}{|c|}{ Estimativas } & \multicolumn{3}{|c|}{ Erro Padrão } & \multirow[t]{2}{*}{$\begin{array}{c}\mathrm{KS} \\
(p-\text { valor })\end{array}$} \\
\hline & & $\hat{\mu}$ & $\hat{\sigma}$ & $\hat{\xi}$ & $\hat{\mu}$ & $\hat{\sigma}$ & $\hat{\xi}$ & \\
\hline Janeiro & GVE & 37,061 & 1,392 & $-0,308$ & 0,223 & 0,155 & 0,076 & 0,707 \\
\hline Fevereiro & GVE & 35,927 & 1,818 & $-0,401$ & 0,295 & 0,211 & 0,090 & 0,580 \\
\hline Março & Gumbel & 34,428 & 1,619 & 0,000 & 0,255 & 0,186 & 0,000 & 0,801 \\
\hline Abril & GVE & 32,013 & 1,983 & $-0,492$ & 0,324 & 0,241 & 0,092 & 0,981 \\
\hline Maio & GVE & 28,648 & 2,189 & $-0,361$ & 0,356 & 0,260 & 0,099 & 0,843 \\
\hline Junho & GVE & 26,732 & 1,827 & $-0,378$ & 0,289 & 0,193 & 0,060 & 0,903 \\
\hline Julho & GVE & 27,465 & 2,138 & $-0,515$ & 0,344 & 0,248 & 0,073 & 0,669 \\
\hline Agosto & Gumbel & 29,264 & 2,485 & $-0,273$ & 0,421 & 0,306 & 0,116 & 0,896 \\
\hline Setembro & GVE & 30,991 & 2,665 & $-0,389$ & 0,437 & 0,316 & 0,099 & 0,976 \\
\hline Outubro & Gumbel & 32,664 & 1,456 & 0,000 & 0,230 & 0,165 & 0,000 & 0,983 \\
\hline Novembro & Gumbel & 34,197 & 1,736 & 0,000 & 0,274 & 0,198 & 0,000 & 0,381 \\
\hline Dezembro & GVE & 36,120 & 1,846 & $-0,323$ & 0,301 & 0,208 & 0,089 & 0,997 \\
\hline
\end{tabular}

Fonte: Autoria própria, 2019.

Nota: $p$-valor $<\alpha$, rejeita-se $H_{0}$, em que $\alpha$ denota o nível de significância do teste.

Podem-se analisar por meio da Tabela 2 os valores de estimativa do parâmetro de forma $\hat{\xi}$, que sugere qual das distribuições (Gumbel, GVE) melhor se ajusta aos dados do referido mês. Desta forma, observa-se que o parâmetro de forma apresentou valores próximos de zero na maioria dos meses analisados, indicando melhor ajuste a distribuição GVE, excetuandose março, outubro, agosto e novembro. Pelo teste de razão de verossimilhança, cuja hipótese $\left(H_{0}\right)$ é de que o parâmetro de forma da distribuição GVE é igual a zero, conclui-se que 
o parâmetro de forma é estatisticamente nulo nos meses de março, outubro e novembro, logo a distribuição Gumbel apresentou melhor ajuste aos dados nesses meses (Tabela 3).

Tabela 3. Resultados do teste de Razão de Verossimilhança para as séries de máximos mensais de Uruguaiana, RS.

\begin{tabular}{cccc}
\hline Mês & $(p$-valor $)$ & Mês & $(p$-valor $)$ \\
\hline Janeiro & $<0,0001$ & Julho & $<0,0001$ \\
Fevereiro & 0,0001 & Agosto & 0,0046 \\
Março & 0,1592 & Setembro & 0,0009 \\
Abril & $<0,0001$ & Outubro & 0,1588 \\
Maio & $<0,0001$ & Novembro & 0,0718 \\
Junho & $<0,0001$ & Dezembro & $<0,0001$ \\
\hline
\end{tabular}

Fonte: Autoria própria, 2019.

Nota: $p$-valor $<\alpha$, rejeita-se $H_{0}$, em que $\alpha$ denota o nível de significância do teste.

A distribuição GVE apresentou melhor ajuste para a maioria dos meses, resultado alinhado com os resultados da literatura. A distribuição GVE também foi o melhor ajuste, e mais comum, na pesquisa de Alam et al. (2018). Dependendo da variável a ser analisada e o tipo de valor extremo avaliado, a distribuição de probabilidade pode sofrer variações, como apresentam Carlos et al. (2017), que concluíram que a distribuição Gumbel se ajustou em alguns meses para a temperatura mínima em Piracicaba-SP.

Após definidas as distribuições aplicadas aos dados, pode-se determinar a probabilidade de ocorrência de uma temperatura superior a $30^{\circ} \mathrm{C}, 35^{\circ} \mathrm{C}, 37^{\circ} \mathrm{C}, 39^{\circ} \mathrm{C}, 40^{\circ} \mathrm{C}, 41^{\circ} \mathrm{C}, 42^{\circ} \mathrm{C}$ e $45^{\circ} \mathrm{C}$. Observa-se que as menores probabilidades de ocorrência de temperaturas altas são nos meses de abril, maio, junho e julho. Essas menores probabilidades ocorrem devido às variações de energia solar entre os solstícios e os equinócios, bem como a atuação das massas de ar sobre a região (SARTORI, 2003). Nos meses de outubro, novembro, dezembro e janeiro as temperaturas altas ocorrem com mais frequência (Tabela 4). Uma interpretação da Tabela 4 pode ser feita da seguinte forma: a probabilidade de que ocorra uma temperatura máxima superior a $40^{\circ} \mathrm{C}$ no mês de janeiro é de $3,26 \%$.

Tabela 4. Probabilidades de ocorrência de temperaturas máximas superiores a $30^{\circ} \mathrm{C}, 35^{\circ} \mathrm{C}, 37^{\circ} \mathrm{C}, 39^{\circ} \mathrm{C}$, $40^{\circ} \mathrm{C}, 42^{\circ} \mathrm{C}$ e $45^{\circ} \mathrm{C}$, em porcentagem (\%) em Uruguaiana, RS.

\begin{tabular}{llllllllll}
\hline \multirow{2}{*}{ Meses } & \multirow{8}{*}{ Distribuição } & \multicolumn{7}{c}{ Temperaturas } \\
\cline { 3 - 9 } & & $30^{\circ} \mathrm{C}$ & $35^{\circ} \mathrm{C}$ & $37^{\circ} \mathrm{C}$ & $39^{\circ} \mathrm{C}$ & $40^{\circ} \mathrm{C}$ & $41^{\circ} \mathrm{C}$ & $42^{\circ} \mathrm{C}$ & $45^{\circ} \mathrm{C}$ \\
\hline Janeiro & GVE & 100 & 96,62 & 64,81 & 14,98 & 3,26 & 0,13 & $<0,01$ & $<0,01$ \\
Fevereiro & GVE & 99,96 & 79,59 & 39,89 & 5,50 & 0,23 & $<0,01$ & $<0,01$ & $<0,01$ \\
Março & Gumbel & 100 & 50,45 & 18,47 & 5,76 & 3,15 & 1,71 & 0,93 & 0,15 \\
Abril & GVE & 89,75 & 6,21 & $<0,01$ & $<0,01$ & $<0,01$ & $<0,01$ & $<0,01$ & $<0,01$ \\
Maio & GVE & 39,18 & $<0,01$ & $<0,01$ & $<0,01$ & $<0,01$ & $<0,01$ & $<0,01$ & $<0,01$ \\
Junho & GVE & 4,95 & $<0,01$ & $<0,01$ & $<0,01$ & $<0,01$ & $<0,01$ & $<0,01$ & $<0,01$ \\
Julho & GVE & 14,79 & $<0,01$ & $<0,01$ & $<0,01$ & $<0,01$ & $<0,01$ & $<0,01$ & $<0,01$ \\
Agosto & GVE & 52,03 & 2,57 & $<0,01$ & $<0,01$ & $<0,01$ & $<0,01$ & $<0,01$ & $<0,01$
\end{tabular}


ANÁLISE PROBABILÍSTICA DA TEMPERATURA MÁXIMA EM URUGUAIANA, RS

\begin{tabular}{llllllllll} 
Setembro & GVE & 75,71 & 9,88 & 0,45 & $<0,01$ & $<0,01$ & $<0,01$ & $<0,01$ & $<0,01$ \\
Outubro & Gumbel & 99,80 & 18,22 & 4,97 & 1,28 & 0,65 & 0,33 & 0,16 & 0,02 \\
Novembro & Gumbel & 100 & 46,72 & 18,04 & 6,09 & 3,47 & 1,97 & 1,11 & 0,20 \\
Dezembro & GVE & 99,99 & 82,46 & 44,90 & 10,80 & 2,93 & 0,26 & $<0,01$ & $<0,01$ \\
\hline
\end{tabular}

Fonte: Autoria própria, 2019.

Apresentam-se na Tabela 5 os níveis de retorno de ocorrência das temperaturas máximas, a partir de diversos períodos de retorno.

Uma interpretação das informações contidas na Tabela 5 pode ser feita do seguinte modo: escolhendo o mês de janeiro e considerando os resultados da distribuição GVE para o tempo de retorno de 10 anos, espera-se que em um tempo médio de 10 anos ocorra pelo menos uma temperatura máxima maior ou igual a $40,19^{\circ} \mathrm{C}$. Pode-se verificar também na Tabela 5 que nos meses de junho, julho e agosto são esperados os menores níveis de temperatura máxima em Uruguaiana-RS. Estes ajustes podem ser utilizados com bastante confiabilidade para extrapolação em períodos mais longos, desde que não ocorram futuras variações climáticas significativas na região (SANSIGOLO, 2008).

Tabela 5. Tempo de retorno de temperatura máxima, em ${ }^{\circ} \mathrm{C}$, de $2,5,10,30,50$ e 100 anos para o município de Uruguaiana-RS.

\begin{tabular}{cccccccc}
\hline Mês & Distribuição & 2 anos & 5 anos & 10 anos & 30 anos & 50 anos & 100 anos \\
\hline Janeiro & GVE & 37,57 & 39,14 & 40,19 & 41,77 & 42,49 & 43,46 \\
Fevereiro & GVE & 36,59 & 38,65 & 40,01 & 42,08 & 43,02 & 44,29 \\
Março & Gumbel & 35,02 & 36,85 & 38,07 & 39,90 & 40,74 & 41,87 \\
Abril & GVE & 32,73 & 34,98 & 36,47 & 38,72 & 39,75 & 41,13 \\
Maio & GVE & 29,45 & 31,93 & 33,57 & 36,05 & 37,19 & 38,72 \\
Junho & GVE & 27,40 & 29,47 & 30,84 & 32,91 & 33,86 & 35,13 \\
Julho & GVE & 28,24 & 30,67 & 32,27 & 34,69 & 35,80 & 37,29 \\
Agosto & GVE & 30,17 & 32,99 & 34,85 & 37,67 & 38,96 & 40,69 \\
Setembro & GVE & 31,96 & 34,98 & 36,98 & 40,00 & 41,38 & 43,25 \\
Outubro & Gumbel & 33,19 & 34,84 & 35,94 & 37,59 & 38,34 & 39,36 \\
Novembro & Gumbel & 34,83 & 36,80 & 38,10 & 40,07 & 40,97 & 42,18 \\
Dezembro & GVE & 36,79 & 38,88 & 40,27 & 42,36 & 43,32 & 44,61 \\
\hline
\end{tabular}

Fonte: Autoria própria, 2019.

Vale ressaltar que a temperatura apresenta relação com outras variáveis meteorológicas (BOMVENTI et al., 2006). Santos e Fisch (2016) verificaram que, baseando-se em uma série de 29 anos da cidade de Taubaté, SP, os resultados para as simulações climáticas da temperatura média anual do ar aumentou em $3,6^{\circ} \mathrm{C}$ e um aumento de $231 \mathrm{~mm}$ na precipitação acumulada para a escala de tempo anual. Righi e Robaina (2010) analisaram as enchentes do rio Uruguai, um dos mais importantes do Rio Grande do Sul, com a ocorrência de precipitações pluviométricas em vários municípios do RS, como Uruguaiana e São Borja, por exemplo, e, de caráter descritivo, verificaram que existe relação entre essas duas variáveis. No entanto, não 
realizaram uma análise estatística que viabilizasse extrapolações de longa duração.

\section{CONCLUSÕES}

Para o município de Uruguaiana-RS, a distribuição generalizada de valores extremos é adequada para estudar o comportamento da temperatura máxima nos meses de janeiro, fevereiro, abril, maio, junho, julho, agosto, setembro e dezembro, e para os meses de março, outubro e novembro a distribuição Gumbel é adequada.

Observa-se que nos meses de maio a agosto ocorreram as menores temperaturas previstas para todos os níveis de retorno, e nos meses de dezembro a fevereiro ocorreram as maiores temperaturas previstas para todos os níveis de retorno calculados.

\section{AGRADECIMENTOS}

Agradecimentos ao BDMET, Banco de Dados Meteorológicos para Ensino e Pesquisa, pela disponibilização da série histórica diária dos dados de temperatura da cidade de Utuguaiana, RS, e à FAPERGS, Fundo de Amparo à Pesquisa do Estado do Rio Grande do Sul, pela concessão da bolsa de iniciação científica.

\section{REFERÊNCIAS BIBLIOGRÁFICAS}

ALAM, A.; EMURA, K.; FARNHAM, C.; YUAN, J. Best-Fit Probability Distributions and Return Periods for Maximum Monthly Rainfall in Bangladesh. Climate, v. 6, n. 9; p. $1-16$, 2018. https://doi.org/10.3390/cli6010009

ARAÚJO, E. M.; SILVA, I. N.; OLIVEIRA, J. B.; JUNIOR, E. G. C.; ALMEIDA, B. M. Evaluation of six distributions of probability at maximum temperature series in Iguatu - CE, Brazil. Revista Ciência Agronômica, v. 41, n. 1, p. 36-45, 2010. https://doi.org/10.5935/1806-6690.20100005

ARTAXO, P. Uma nova era geológica em nosso planeta: Antropoceno? Revista USP, n. 103, p. 13-24, 2014. https: // doi. org / 10. 11606 / issn . 23 16- 9036.v0i103p13-24

ASTOLPHO, F.; CAMARGO, M. B. P. DE; BARDIN, L. Probabilidades mensais e anuais de ocorrência de temperaturas mínimas do ar adversas à agricultura na região de Campinas (SP), de 1891 a 2000. Bragantia, v. 63, n. 1, p. 141-147, 2004. https://doi.org/10.1590/S000687052004000100014

AYOADE, J. O. Introdução à climatologia para os trópicos. 11. ed. Rio de Janeiro: Bertrand Brasil, 2006. 350 p.

BAUTISTA, E. A. L.; ZOCCHI, S. S.; ANGELOCCI, L. R. Fitting the generalized extreme value distribution (GEV) to the maximum wind speed data in Piracicaba, São Paulo, Brazil. Revista Matemática e Estatística, v. 22, n. 1, p. 95-111, 2004.

BEIJO, L. A.; AVELAR, F. G. Distribuição generalizada de valores extremos no estudo de dados climáticos uma breve revisão e aplicação. Revista da Estatística da Universidade Federal de Ouro Preto, v. 1, n. 1, p. 10-16, 2011.

BOMVENTI, T. N.; WAINER, I. E. K. C.; TASCHETTO, A. S. Relação entre a radiação de onda longa, precipitação e temperatura da superfície do mar no oceano atlântico tropical. Revista Brasileira de Geofísica, v. 24, n. 4, p. 513-524, 2006. https://doi.org/10.1590/S0102261X2006000400005

CAMARGO, M. B. P. DE; JUNIOR, M. J. P.; ALFONSI, R. R.; ORTOLANI, A. A.; BRUNINI, O. Probabilidade de ocorrência de temperaturas mínimas absolutas mensais e anual no estado de São Paulo. Bragantia, v. 52, n. $\quad 2, \quad$ p. 161-168, 1993. http://doi.org/10.1590/S000687051993000200008

CARGNELUTTI FILHO, A.; MALUF, J. R. T.; MATZENAUER, R.; STOLZ, A. P. Altitude and geographic coordinates in the tenday mean minimum air temperature estimation in the State of Rio Grande do Sul, Brazil. 
Pesquisa Agropecuaria Brasileira, v. 41, n. 6, p. 893-901, 2006. https://doi.org/10.1590/S0100204X200600060000

COLES, S. An Introduction to Statistical Modeling of Extreme Values. London: Springer London, 2001. 221 p. https://doi.org/10.1007/978-1-4471-3675-0

COSTA, L.; SEDIYAMA, G. C. Elementos climáticos e produtividade agrícola. Ação ambiental, v. 2, n. 7, p. 24-27, 1999.

GILLELAND, E.; KATZ, R. W. extRemes 2.0: An Extreme Value Analysis Package in $R$. Journal of Statistical Software, 2016. https://doi.org/10.18637/jss.v072.i08

HARTMANN, M.; MOALA, F. A.; MENDONÇA, M. A. Estudo das precipitações máximas anuais em Presidente Prudente. Revista Brasileira de Meteorologia, v. 26, $\mathrm{n}$. 4, p. 561-568, 2011. https://doi.org/10.1590/S0102-

77862011000400006

INMET - Instituto Nacional de Meteorologia. BDMEP - Banco de Dados Meteorológicos para Ensino e Pesquisa. Dados climatológicos da estação 83927 - Uruguaiana - RS. Disponível em: http://www.inmet.gov.br/portal/index.php?r=b dmep/bdmep. Acesso em: 10 abr. 2018.

JENKINSON, A. F. The frequency distribution of the annual maximum (or minimum) values of meteorological elements. Quarterly Journal of the Royal Meteorological Society, v. 81, n. 348, p. 158-171, 1955. https://doi.org/10.1002/qj.49708134804

QUADROS, L. E. DE; QUEIROZ, M. M. F. DE; BOAS, M. A. V. Distribuição de frequência e temporal de chuvas intensas. Acta Scientiarum Agronomy, v. 33, n. 3, 2011. https://doi.org/10.4025/actasciagron.v33i3.602 1

R CORE TEAM. R: A language and environment for statistical computing. ISBN 3-900051-07-0

REIS, C. J. DOS; BEIJO, L. A.; AVELAR, F. G. Temperatura mínima esperada para Piracicaba-SP via distribuições de valores extremos. Revista Brasileira de Agricultura Irrigada, v. 11, n. 4, p. 1639-1650, 2017. https://doi.org/10.7127/rbai.v11n400574

RIGHI, E.; ROBAINA, L. E. DE S. Enchentes do Rio Uruguai no Rio Grande do Sul entre 1980 e 2005: uma análise geográfica. Sociedade \& Natureza, v. 22 , n. 1, p. 35-54, $2010 . \quad$ https://doi.org/10.1590/S198245132010000100004

SALVIANO, M. F.; GROPPO, J. D.; PELLEGRINO, G. Q. Análise de Tendências em Dados de Precipitação e Temperatura no Brasil. Revista Brasileira de Meteorologia, v. 31, n. 1, p. 64-73, 2016. http://doi.org/10.1590/0102-77862015000

SANSIGOLO, C. A. Distribuições de extremos de precipitação diária, temperatura máxima e mínima e velocidade do vento em Piracicaba, SP (1917-2006). Revista Brasileira de Meteorologia, v. 23, n. 3, p. 341-346, 2008. https://doi.org/10.1590/S010277862008000300009

SANTOS, T. A.; FISCH, G. Temperatura e precipitação: futuros cenários do município de Taubaté, SP, Brasil. Ambiente e Agua - An Interdisciplinary Journal of Applied Science, v. 11, n. 5, p. 1068-1087, 2016. https://doi.org/10.4136/ambi-agua.1896

SARTORI, A. A. DA C.; SILVA, A. F.; RAMOS, C. M. C.; ZIMBACK, C. R. L. Variabilidade temporal e mapeamento dos dados climáticos de Botucatu - SP. IRRIGA, v. $15, \quad$ n. $2, \quad$ p. 131-139, 2010. https://doi.org/10.15809/irriga.2010v15n2p13 1

SARTORI, M. G. B. A dinâmica do clima do Rio Grande do Sul: indução empírica e conhecimento científico. Revista Terra Livre, 
Ferreira et al.

v. 20, p. 27-50, 2003

STHEPHENSON, A. G. evd: Extreme Value Distributions. R News, v. 2, n. 2, p. 31-32, 2002.

THOMAS, M.; LEMAITRE, M.; WILSON, M. L.; VIBOUD, C.; YORDANOV, Y.; WACKERNAGEL, H.; CARRAT, F. Applications of Extreme Value Theory in Public Health. PLOS ONE, v. 11, n. 7, p. 1-7, 2016.https://doi.org/10.1371/journal.pone.015 9312

WALTER, L. C.; STRECK, N. A.; ROSA, H. T.; FERRAZ, S. E. T.; CERA, J. C. Mudanças climáticas e seus efeitos no rendimento de arroz irrigado no Rio Grande do Sul. Pesquisa Agropecuária Brasileira, v. 49, n. 12 , p. 915-924, $2014 . \quad$ https://doi.org/10.1590/S0100204X2014001200001 